\title{
Novel Surgical Technique for Bone Marrow Lesion - Case Report
}

\author{
Pal Fodor', Radu Prejbeanu², Vlad Predescu3 , Bogdan Codorean³ ${ }^{3}$ Radu Fleaca4 ${ }^{4}$, Mihai Roman", \\ Adrian Todor ${ }^{5}$, Octav Russu', Tiberiu Bățagă ${ }^{1}$ \\ ${ }^{1}$ University of Medicine and Pharmacy, Tîrgu Mureș, Romania \\ 2 "Victor Babeș" University of Medicine and Pharmacy, Timișoara, Romania \\ 3 "Carol Davila" University of Medicine and Pharmacy, Bucharest, Romania \\ 4 "Victor Papillan" Faculty of Medicine, "Lucian Blaga" University, Sibiu, Romania \\ 5 "Iuliu Hațieganu" University of Medicine and Pharmacy, Cluj-Napoca, Romania
}

\section{CORRESPONDENCE}

\section{Pal Fodor}

Str. Gheorghe Marinescu nr. 38 540139 Tîrgu Mureș, Romania

Tel: +40 265215551

E-mail: fodorpaly22@gmail.com
Radu Prejbeanu • P-ța Eftimie Murgu nr. 2, 300041 Timișoara, Romania. Tel: +40 256204 400, E-mail: raduprejbeanu@gmail.com

Vlad Predescu • B-dul Eroii Sanitari nr. 8, 050474 București, Romania. Tel: +40 213180 719, E-mail: vladpredescu001@gmail.com

Bogdan Codorean • B-dul Eroii Sanitari nr. 8, 050474 București, Romania. Tel: +40 213180 719, E-mail: dr.codorean@traumatologie-sportiva.ro

Radu Fleaca • Str. Lucian Blaga nr. 2A, 550169 Sibiu, Romania. Tel: +40 269212 320, E-mail: rfleaca@ yahoo.com

Mihai Roman • Str. Lucian Blaga nr. 2A, 550169 Sibiu, Romania. Tel: +40 269212 320, E-mail: mihaidanroman@yahoo.com

Adrian Todor • Str. Emil Isac nr. 13, 400023 ClujNapoca, Romania. Tel: +40 264406 843, E-mail: adi. todor@yahoo.com

Octav Russu • Str. Gheorghe Marinescu nr. 38 , 540139 Tîrgu Mureș, Romania. Tel: +40 265215 551, E-mail:octav@genunchi.ro

Tiberiu Bățagă • Str. Gheorghe Marinescu nr. 38, 540139 Tîrgu Mureș, Romania. Tel: +40 265215551 E-mail: tbataga@gmail.com

\begin{abstract}
Introduction: Bone marrow lesions (BMLs) are commonly described as magnetic resonance imaging (MRI) findings associated with stress injuries or trauma. The presence of BMLs closely correlates with pain and rapid joint deterioration. Case presentation: A 51-year-old healthy man presented to our clinic with severe knee pain due to BMLs. After 3 months of conservative treatment, arthroscopy and subchondroplasty (SCP) of the medial femoral condyle was performed. The IKDC (International Knee Documentation Committee) score improved from 39.9 to 66.7 at 6 months, and to 87.4 at 1 year after surgery. The KOOS (Knee Injury and Osteoarthritis Outcome) score improved from 38.5 to 77.7 at 6 months, and to 92.6 at 1 year after surgery. The Tegner Lysholm score improved from 23 to 80 at 6 months, and to 95 at 1 year after surgery. Conclusion: SCP may provide a viable approach to reduce pain associated with BML, with minimal risk of significant complications.
\end{abstract}

Keywords: bone marrow lesion, subchondral bone, subchondroplasty, cartilage, osteoarthritis

\section{INTRODUCTION}

Primary osteoarthritis, the most common disease of the joints, is characterized by a multifactorial pathogenesis involving the articular cartilage, synovium, subchondral bone and bone marrow, menisci, ligaments and supporting musculature. ${ }^{1}$ The biomechanical relationships between the articular cartilage and the underlying subchondral bone play a significant role in the initiation and progression of osteoarthritis. ${ }^{2}$

Recent studies show that cartilage and subchondral bone act as a single functional unit (osteochondral unit), which absorbs and distributes loads across the joint. Changes in one component may affect all other components. ${ }^{3}$

Changes in the subchondral bone, termed bone marrow lesions (BMLs), are commonly described as magnetic resonance imaging (MRI) findings associat- 
ed with stress injuries or trauma. ${ }^{4}$ In fat-suppressed MRI sequences including T2 Fat Sat, Proton Density (PD) Fat Sat, or short TI inversion recovery, they appear as diffuse water-consistent signals in the marrow space. ${ }^{4,5}$ The presence of BMLs has been shown to correlate strongly with the presence of pain (presence and severity) and rapid joint deterioration. ${ }^{4}$

Histologically, BMLs demonstrate fibrosis, osteonecrosis, increased subchondral vascularization and extensive bony remodeling, and are likely the result of contusions and focal microfractures. ${ }^{5}$ Patients with BMLs have been shown to have more rapid cartilage destruction and subchondral bone attrition. ${ }^{5-7}$

\section{CASE PRESENTATION}

A 51-year-old healthy man presented to our clinic with severe knee pain. He was treated for 3 months with nonsteroidal anti-inflammatory drugs, physiotherapy and non-weight bearing, but the symptoms did not resolve. The patient agreed to the publication of his data and the institution where the patient had been admitted, approved the publication of the case.

\section{Investigations}

Range of motion in the knee was markedly limited with an arc of 15-90, with no malalignment in the affected lower limb. On plain radiographs he had Kellgren-Lawrence grade 2 knee osteoarthritis.

On MRI, subchondral bone marrow signal alterations were present in the medial femoral condyle. On T2 (T2w) - or proton density-weighted (PDw), fat suppressed (FS), fast spin echo (FSE) or short tau inversion recovery (STIR) images he had ill-defined subchondral areas of high signal intensity. On the basis of these clinical and imaging investigations we reached to the diagnosis of bone marrow lesions (BML) (Figure 1).

\section{Treatment}

After 3 months of conservative treatment, we decided on surgical intervention, and performed arthroscopy and subchondroplasty of the medial femoral condyle under general anesthesia. The patient was placed in supine position on a radiolucent operating table.

At standard arthroscopic evaluation of the knee we found grade II ICRS cartilage lesions on the medial femoral condyle. The medial and lateral meniscus, and the anterior and posterior cruciate ligaments were probed for

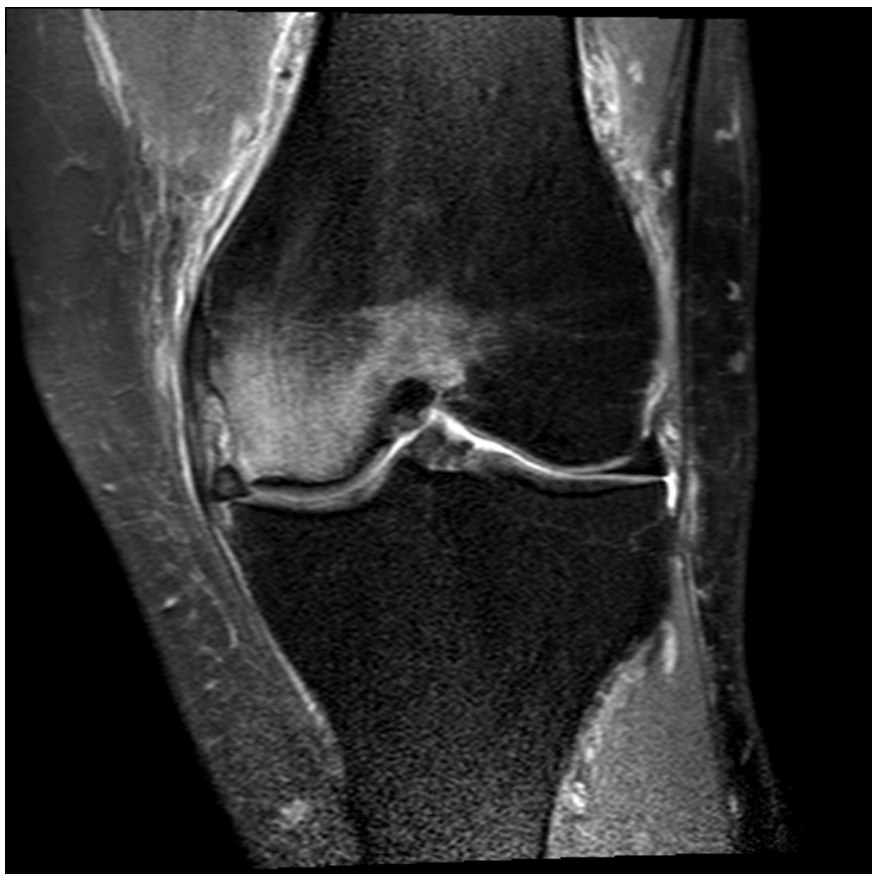

FIGURE 1. MRI subchondral bone marrow signal alterations present in the medial femoral condyle.

stability. We performed a debridement chondroplasty for loose chondral flaps.

Once the arthroscopy was completed, we prepared the operative leg for the subchondroplasty procedure. The leg was elevated to obtain a lateral and anterior-posterior fluoroscopic view. We placed the guide wire by a trajectory that was predetermined by 3 MRI planes (axial, coronal and sagittal). The cannula placement was confirmed on orthogonal fluoroscopic views. The bone substituent (Wright, Prodense Bone Substitute) was injected into the lesion.

\section{Rehabilitation}

The patient was non-weight bearing for 14 days. Physical therapy was initiated 14 days after surgery, and a return to full, unrestricted activities was allowed 4 weeks postoperatively.

We augmented our procedure with ACP (Autologous Conditioned Plasma, Arthrex ACP system) injections. We started the plasma therapy on the $14^{\text {th }}$ day after surgery, and we performed 5 injections with ACP (Autologous Conditioned Plasma), once every 7 days.

\section{Outcome and follow-up}

The clinical outcome (pain, function, and quality of life) was assessed using the International Knee Documentation 


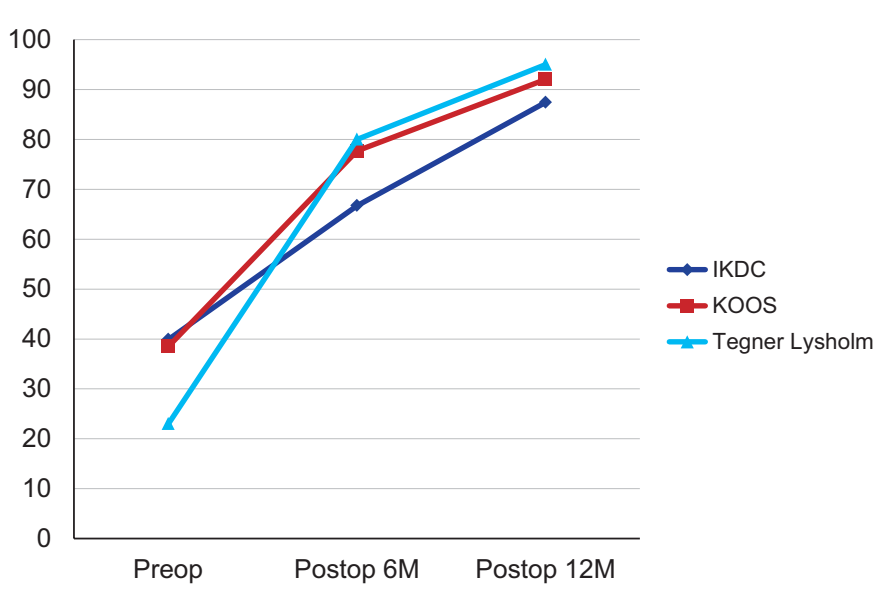

FIGURE 2. Clinical outcome assessed using the IKDC score, the KOOS, and the Tegner Lysholm score, recorded prior to the surgical intervention, and then at 6 months and at one year.

Committee Subjective Knee Form (IKDC), the Knee Injury and Osteoarthritis Outcome Score (KOOS), and the Tegner Lysholm score, recorded prior to the surgical intervention, and then at 6 months and at one year.

The IKDC score improved from 39.9 to 66.7 at 6 months, and to 87.4 at 1 year after surgery (Figure 2). The KOOS score improved from 38.5 to 77.7 at 6 months, and to 92.6 at 1 year after surgery (Figure 2). The Tegner Lysholm score improved from 23 to 80 at 6 months, and to 95 at 1 year after surgery (Figure 2). The postoperative range of motion also improved with an $\operatorname{arc}$ of $0^{\circ}$ to $120^{\circ}$.

\section{DISCUSSION}

An observational study of patients with osteoarthritis found that compared with patients without BMLs, patients with MRI-observed BMLs were nearly nine times as likely to progress to TKA over a 3-year follow-up period. ${ }^{8}$ Also, Wluka et al. suggested that BMLs, present in healthy asymptomatic individuals with no history of significant knee pain or trauma, are associated with increased risk of cartilage defect progression and loss of cartilage volume. ${ }^{7}$ This suggests that an intervention with the capacity to repair subchondral bone, and interfere with rapid joint deterioration associated with a BML, may be an important preventive measure for knee osteoarthritis.

Several arthroscopic and open surgical techniques have been used to address subchondral disease, including: osteochondral autograft and allograft, marrow-stimulation techniques, autologous chondrocyte implantation via sandwich technique, unloading osteotomy, arthroplasty, and subchondroplasty (Zimmer Knee Creations). ${ }^{2}$
As our patient was an active 51-year-old man who clearly expressed his wish to return to work as soon as possible, we opted for a joint-preserving surgical technique termed subchondroplasty (SCP). It represents a minimally invasive method with the capacity to relieve symptoms, and enables patients to resume certain normal activities as soon as 2 weeks postprocedure. ${ }^{2}$ SCP is performed under fluoroscopic guidance by injecting a flowable osteoconductive bone void filler (calcium phosphate) into the subchondral bone that corresponds to the area of bone marrow edema on MRI. The injected bone substitute stabilizes the mechanical insufficiency of the subchondral bone, providing pain relief. ${ }^{4}$ The bone substitute is resorbed over time, and induces the restoration of the structural integrity of the damaged bone. ${ }^{5}$

The treatment of subchondral bone marrow lesions by means of subchondroplasty has limited yet promising short-term clinical outcomes. Up to date there are few published results in the literature, with a limited number of patients enrolled. $4,9,10$

Recent studies have shown that platelet-rich plasma may be helpful for the treatment of osteoarthritis by means of conservative intra-articular delivery. ${ }^{11}$ Platelet-rich plasma is thought to stimulate the proliferation of chondrocytes and the differentiation of mesenchymal cells of the subchondral bone into the chondrogenic line. ${ }^{12}$ This was the rationale underlying our decision to combine of subchondroplasty with plasma injections.

After one year of follow-up, a good result was obtained in this case, with a relatively short recovery time.

\section{CONCLUSION}

Subchondroplasty may provide a viable approach to reduce pain associated with BMLs, with minimal risk of significant complications. It represents a less invasive, jointpreserving option in patients with BMLs.

\section{CONFLICT OF INTEREST}

Nothing to declare.

\section{REFERENCES}

1. Farr J, Cohen SB. Expanding Applications of the Subchondroplasty Procedure for the Treatment of Bone Marrow Lesions Observed on Magnetic Resonance Imaging. Operative Techniques in Sports Medicine. 2013;21:138-143.

2. Geoffrey D. Abrams, Joshua D. Harris, Brian J. Cole. Subchondral Bone Treatment. Cole BJ, Harris JD, eds. Biologic Knee Reconstruction: A Surgeon's Guide (pp 83-89). (C) 2015 SLACK Incorporated.

3. Mahjoub M, Berenbaum F, Houard X. Why subchondral bone in osteoarthritis? The importance of the cartilage bone interface in osteoarthritis. Osteoporos Int. 2012;23(Suppl8):S841-S846. 
4. Cohen SB, Sharkey PF. Subchondroplasty for Treating Bone Marrow Lesions. J Knee Surg. 2016;29:555-563.

5. Cohen SB, Sharkey PF. Surgical Treatment of Osteoarthritis Pain Related to Subchondral Bone Defects or Bone Marrow Lesions: Subchondroplasty. Techniques in Knee Surgery. 2012;11:170-175.

6. Roemer FW, Neogi T, Nevitt MC, et al. Subchondral bone marrow lesions are highly associated with, and predict subchondral bone attrition longitudinally: the MOST study. Osteoarthritis Cartilage. 2010:18:47-53.

7. Wluka AE, Wang Y, Davies-Tuck M, English DR, Giles GG, Cicuttini FM. Bone marrow lesions predict progression of cartilage defects and loss of cartilage volume in healthy middle-aged adults without knee pain over 2 yrs. Rheumatology (Oxford). 2008;47:1392-1396.
8. Scher C, Craig J, Nelson F. Bone marrow edema in the knee in osteoarthrosis and association with total knee arthroplasty within a threeyear follow-up. Skeletal Radiol. 2008;37:609-617.

9. Abrams GD, Alentorn-Geli E, Harris JD, Cole BJ. Treatment of a lateral tibial plateau osteochondritis dissecans lesion with subchondral injection of calcium phosphate. Arthrosc Tech. 2013;2:e271-274.

10. Sharkey PF, Cohen SB, Leinberry CF, Parvizi J. Subchondral bone marrow lesions associated with knee osteoarthritis. Am J Orthop (Belle Mead NJ). 2012:41:413-417.

11. Marmotti A, Rossi R, Castoldi F, Roveda E, Michielon G, Peretti GM. RP and Articular Cartilage: A Clinical Update. Biomed Res Int. 2015;2015:542502.

12. Manunta AF, Manconi A. The treatment of chondral lesions of the knee with the microfracture technique and platelet-rich plasma. Joints. 2013;1:167-170. 